\title{
Fatores de risco para o parto prematuro em uma maternidade estadual de referência
}

\section{Risk factors for preterm birth in a state seminal maternity hospital}

\author{
Bruna Fernanda Almeida, Ananda Madeira Moura Fé Aguiar, Tassio Breno De Sousa \\ Lopes Lavôr, Maria Eliane Martins Oliveira Da Rocha, Kleiton Richard Da Silva Araújo
}

RESUMO

Objetivo: Analisar a presença de fatores de risco para o parto prematuro em puérperas de uma maternidade Estadual de referência. Métodos: A pesquisa é um estudo de campo de natureza exploratória descritiva, com abordagem quantitativa. Sendo desenvolvida em uma Maternidade Estadual de referência situada no município de Teresina-PI na qual teve como participantes da pesquisa 57 puérperas que tiveram seus partos prematuros entre os meses de abril e maio de 2014. A coleta de dados ocorreu após aprovação do Comitê de Ética em Pesquisa, através de informações obtidas em entrevistas com as puérperas, além da avaliação dos registros sobre seus partos, contidos nos prontuários. Posteriormente os mesmos foram analisados, digitados e tabulados no Microsoft Excel 2010, que se configura como uma ferramenta de tratamento de dados e análise estatística. Resultados: Quanto ao perfil: 35,9\% tinham entre 19 e 25 anos de idade, $78,94 \%$ eram casadas ou em união estável, 26,30\% ensino fundamental incompleto, 50,90\% eram trabalhadoras do lar e 59,60\% tinham renda de 1 a 3 salários mínimos; quanto a historia clinica: $61,40 \%$ eram primíparas, $12,28 \%$ já tinham tido parto prematuro prévio, e aborto prévio 14,03\%, tiveram hipertensão arterial sistêmica durante a gestação $36,84 \%$, bacteriúria $31,57 \%$, e metrorragia após a $12^{\circ}$ semana de gestação 19,29\%. Observou-se ainda a presença de patologias encontradas com frequência nas puérperas que tiveram seus recém-nascidos prematuros, sendo elas: pré-eclâmpsia, eclâmpsia, oligodramnia, aminiorrexe prematura, descolamento prematuro de placenta e vulvovaginite. Conclusões: O estudo mostrou que o parto prematuro ocorre com frequência entre mulheres jovens, com situação civil estável, que não trabalham fora de casa e com baixa renda. Outro ponto observado foi a presença de parto prematuro anterior ou aborto prévio, além de problemas como bacteriúria, metrorragia, hipertensão arterial sistêmica e outras doenças que poderiam ter tido um acompanhamento melhor, diminuindo os riscos de parto prematuro.

Palavras-chave: Nascimento Prematuro; Fatores de Risco; Enfermagem.

\section{ABSTRACT}

Objective: To analyze the presence of risk factors for premature birth in women from a state seminal Maternity Hospital. Methods: The research is a descriptive exploratory field study within a quantitative approach. It was developed in a state seminal Maternity Hospital located in the city of Teresina, PI. It had as research participants 57 women who had their premature births between the months of April and May in 2014. The data collection took place after approval by the local Ethics in Research Committee, with information obtained through interviews with mothers, besides the records evaluation on their deliveries, contained in their medical records. Afterwards, the data was analyzed, typed, and tabulated in Microsoft Excel 2010, which is a data processing and statistical analysis tool. Results: Regarding the profile: $35.9 \%$ had between 19 and 25 years old; $78.94 \%$ were married in a stable relationship; $26.30 \%$ did not finish Middle School; $50.90 \%$ were house workers, and $59.60 \%$ had incomes three times the minimum wage. Concerning to the clinical history: $61.40 \%$ were primiparous; $12.28 \%$ had already had preview preterm delivery , and $14.03 \%$ had prior abortion; $36.84 \%$ had high blood pressure during pregnancy; $31.57 \%$ had bacteriuria; and $19.29 \%$ had metrorrhagia after the 12 [ week of pregnancy. There was also the presence of pathologies frequently found in mothers who had their newborns premature, such as: pre-eclampsia, eclampsia, oligohydramnious, premature amnniorrexe, premature placenta removal, and vulvovaginitis. Conclusions: The study showed that the preterm birth occurs frequently among young women in stable marital situation, who do not work outside their homes or have low incomes. Another point was the presence of preview preterm birth or prior abortion, besides problems as bacteriuria, metrorrhagia, hypertension, and other diseases that could have had better monitoring, what would reduce the risk of premature birth.

Keywords: Premature Birth, Risk Factors, Nurcing. 


\section{INTRODUÇÃO}

A prematuridade configura-se como umas das maiores causas de mortalidade infantil no Brasil, e está relacionada às condições perinatais, sendo decorrente também de circunstâncias diversas e imprevisíveis, em todos os lugares e classes sociais e acarretam à família e à sociedade em geral um custo social e financeiro de difícil mensuração. Segundo a Organização Mundial de Saúde (OMS), o parto é considerado prematuro quando ocorre antes da $37^{a}$ semana de gestação e entre as causas mais comuns para a prematuridade está à gravidez na adolescência, a ruptura prematura da bolsa, gestações de gêmeos e problemas de saúde da mãe como: pressão arterial alta, infecção urinária, diabetes e tabagismo. A "cesariana de hora marcada" contribui também com o aumento no número de bebês que nascem prematuros ${ }^{1}$.

Esses fatores têm exigido do sistema de saúde a implantação de estratégias que forneçam uma estrutura assistencial, capacidade técnica e equipamentos que nem sempre estão disponíveis. É um problema que afeta diretamente a estrutura familiar alterando as expectativas e anseios que permeiam a perinatalidade, tornando-se difícil avaliar os componentes que influenciam e são influenciados pelo complexo processo do nascimento prematuro. A mortalidade e a morbidade neonatal são maiores entre os neonatos prematuros, além disso, a carga econômica associada a esses nascimentos é significativa, na medida em que o parto prematuro demanda assistência e cuidados de maior nível de complexidade, especialmente com relação ao neonato².

Creasy et al., em São Francisco (EUA), num esforço para identificar aquelas pacientes com risco aumentado para parto prematuro desenvolveram um escore que leva em conta: 1. Status socioeconômico, 2. História prévia, 3. Hábitos maternos e 4. Acontecimentos durante a atual gestação. As pacientes foram avaliadas na sua primeira visita pré-natal e novamente com 25-28 semanas. Aquelas com escore de 10 foram classificadas como de alto risco de parto prematuro. Nessas pacientes de alto risco, 30\% tiveram partos prematuros, contra 2,5\% das pacientes com baixo risco ${ }^{3}$.

No início dos anos 80 foram publicados outros estudos que relatavam os resultados de programas de prevenção da prematuridade (Herron et al., 1982; Papiernik et al., 1985), os quais criaram grandes expectativas de que uma metodologia efetiva para a prevenção deste evento estivesse delineada. Estes trabalhos selecionaram gestantes com risco elevado para a prematuridade, após a aplicação de escores de predição baseados em fatores de risco, deram ênfase aos seguintes aspectos: educação das pacientes e da equipe médica, modificação de certos fatores de risco, redução da atividade física e licença do trabalho assalariado promovido pelo sistema de seguridade, detecção precoce do trabalho de parto antes do termo e intervenção medicamentosa agressiva para inibir o trabalho de parto como processo de intervenção no grupo de estudo4.

O estudo de Papiernik et al., (1985), relataram declínio na proporção de partos prematuros em Haguenau, de $5,4 \%$ para 3,7\%, após vários anos de implementação de um projeto que trabalhou fatores associados à atividade física, com modificação do estilo de vida das pacientes e até mesmo afastamento do trabalho assalariado. Este programa, na França, baseou-se principalmente na modificação dos fatores sociais, demonstrando compensações econômicas no campo da Seguridade Social ${ }^{4}$.

Nos Estados Unidos da América do Norte, Herron et al., 1982, encontraram diminuição significativa da taxa de prematuridade, de 6,75\%, antes da implantação do programa semelhante ao francês na Califórnia, para 2,4\% após um ano. Esse programa, baseado no estudo prévio de Creasy et al., 1980, foi composto pelo rastreamento dependente do risco, processo educacional intensivo para gestantes no que se refere à sintomatologia do parto prematuro, exames pélvicos semanais e internação imediata e abordagem tocolítica precoce quando presentes os sinais do parto antes do termo ${ }^{4}$.

Considera-se nos estudos que a assistência pré-natal tem importância fundamental no rastreamento e diagnóstico das pacientes que vão ter o parto pré-termo, para que haja possibilidade de se tomar medidas que tentem impedi-lo ou que ao menos garantam sobrevida do concepto com a menor morbidade possível ${ }^{5}$.

Observa-se que uma forma viável e eficiente de intervir e prevenir agravos ou riscos é justamente o conhecimento e o monitoramento dos fatores de risco, bem como das condições de nascimento, considerando o estado geral, as condições de saúde da mãe e a assistência prestada no processo do nascimento. Acredita-se que identificar fatores de risco associados ao ciclo gravídico-puerperal possibilita direcionar e adotar medidas preventivas e curativas de forma adequada à realidade. Diante disso, esse estudo teve como objetivo: avaliar a presença de fatores de risco nas mulheres que passaram pelo parto prematuro e que foram assistidas em uma maternidade Estadual de referência. Para 
tanto propôs-se levantar o perfil dessas mulheres/puérperas e verificar a presença dos fatores de risco, utilizando como referência dados do Ministério da Saúde (MS) e da escala de Creasy et al (que trabalha os fatores socioeconômicos, história materna, hábitos maternos e dados da gestação atual), além de investigar quais intervenções assistenciais foram realizadas para gestantes de risco durante o pré-natal, para evitar e/ou minimizar as chances do parto prematuro.

A partir dos resultados da pesquisa pode-se classificar os riscos para gestante de ter um parto prematuro e assim, fornecer dados que orientem as decisões a serem tomadas diante dessa situação, tal como, encaminhamento para unidades hospitalares de referência, como forma de diminuir os riscos nos quais a mãe e recém-nascido estão expostos.

\section{METODOLOGIA}

A pesquisa é um estudo de campo de natureza exploratório descritivo prospectivo, com abordagem quantitativa. Tem como objetivo principal a analise dos fatores de risco para prematuridade.

Estudos descritivos têm como objetivo primordial a descrição das características entre variáveis. E uma de suas características mais significativas está na utilização de técnicas padronizadas de coleta de dados, tais como questionários, formulários e a observação sistemática ${ }^{6}$.

O presente estudo foi desenvolvido em uma Maternidade Estadual de referência situada no município de Teresina, Estado do Piauí. Teve como participantes puérperas que tiveram parto prematuro no período da coleta de dados, compreendida entre abril e maio de 2014. Apresentando como população e amostra o número de todas as puérperas atendidas no período e que se enquadrem nos critérios de inclusão que foram: puérperas que tiveram parto prematuro, bem como a aceitação das mesmas em participar da pesquisa. E sendo utilizado como critério de exclusão da amostra: gemelaridade e gestações com crianças que nascerem com malformações. 0 estudo trabalhou com um total de 57 mulheres que tiveram seus partos prematuros na maternidade de referência Estadual e que se enquadraram nos critérios determinados.

Os dados foram coletados, através de informações obtidas na entrevista realizada com as puérperas e também em registros feitos sobre o parto nos prontuários. Como instrumento de coleta de dados utilizou-se um formulário que contém dados relacionados aos objetivos do estudo e a escala de Escore de Creasy et al.

$O$ instrumento de coleta de dados foi testado e validado, o que possibilitou verificar até que ponto estava claro, compreensivo e se realmente apresentava condições de garantir resultados isentos de erros. $O$ instrumento foi aplicado em entrevistas feitas pelos pesquisadores nos meses de abril e maio de 2014, registradas no formulário e estes, foram complementados pelos dados dos prontuários das participantes, sendo a duração determinada pelo andamento da entrevista.

Após a coleta de dados, os mesmos foram analisados, digitados e tabulados no Microsoft Excel 2010, que se configura como uma ferramenta de tratamento de dados e análise estatística. Na análise destes dados, foram utilizadas estatísticas descritivas, tais como: distribuição de frequências, simples, percentuais. $\mathrm{Na}$ análise da escala de Escore de Creasy et al utilizou-se os escores para avaliar o risco aumentado para o parto prematuro e que foram definidos, determinados e validados por este: 1. Baixo risco - escore de $0-5$ pontos; 2. Médio risco - escore de $6-9$ pontos e 3. Alto risco - escore de 10 ou mais pontos. A discussão dos dados foi feita através do confronto com estudos referenciados sobre tema abordado.

Quanto às questões e aspectos éticos, foram respeitados na pesquisa, pois foi solicitada autorização da referida maternidade por meio do Termo de Compromisso de Utilização de Dados (TCUD) e da Carta de Anuência da Instituição, além da assinatura do Termo de Consentimento Livre e Esclarecido (TCLE), pelas participantes da pesquisa. A busca de dados necessários para o trabalho necessitou, ainda, da aprovação do Comitê de Ética e Pesquisa (CEP) da Universidade Estadual do Piauí (UESPI) via Plataforma Brasil e tem como CAAE n 16600013.4.0000.5209. Uma vez deliberado 0 Parecer Consubstanciado de Aprovação, teve início à coleta de dados, seguindo, portanto as determinações da ética na pesquisa em saúde, conforme Resolução 466/2012 do Conselho Nacional de Saúde (CNS), que determina as normas e diretrizes regulamentadoras de pesquisa com Seres Humanos. 


\section{RESULTADOS}

A Tabela 1 retrata o perfil das 57 mulheres puérperas pesquisadas que tiveram seus filhos prematuros na maternidade.

Tabela 1 - Perfil sócio demográfico das puérperas atendidas na maternidade de referência

Estadual. Teresina-PI, 2014.

\begin{tabular}{lcc}
\hline \multicolumn{1}{c}{ DADOS DAS PUÉRPERAS } & NÚMERO & PORCENTAGEM \\
\hline 1 - IDADE & & \\
12 a 18 anos & 12 & $21,05 \%$ \\
19 a 25 anos & 20 & $35,09 \%$ \\
26 a 32 anos & 14 & $24,56 \%$ \\
33 a 39 anos & 6 & $10,52 \%$ \\
40 anos ou mais & 5 & $8,78 \%$ \\
2- ESTADO CIVIL & & \\
Solteira & 10 & $17,60 \%$ \\
Casada ou união estável & 45 & $78,90 \%$ \\
Separada/divorciada & 2 & $3,50 \%$ \\
3-N DE FILHOS & & \\
1 a 2 & 35 & $61,40 \%$ \\
3 a 4 & 17 & $29,80 \%$ \\
4 ou mais & 5 & $8,80 \%$ \\
4-GRAU DE INSTRUÇÃO & & \\
Ensino fundamental incompleto & 15 & $26,30 \%$ \\
Ensino fundamental completo & 7 & $12,30 \%$ \\
Ensino médio incompleto ou completo & 35 & $61,40 \%$ \\
Ensino superior & 0 & $0 \%$ \\
5-PROFISSÃO & & \\
Do lar & 29 & $50,90 \%$ \\
Estudante & 4 & $7,00 \%$ \\
Lavradora & 9 & $15,80 \%$ \\
Outros & 15 & $26,30 \%$ \\
6- RENDA MENSAL & & \\
Sem renda fixa ou <1 & 20 & $35,00 \%$ \\
De 1 a 3 & 34 & $59,60 \%$ \\
De 4 a 7 & 2 & $3,50 \%$ \\
De 8 ou mais & 1 & $1,80 \%$ \\
\hline
\end{tabular}

Quanto à idade houve predomínio da faixa etária de 19 a 25 anos, correspondendo a 35,09\%, seguida das mulheres com idade de 26 a 32 anos com $24,56 \%$ da amostra. Já no que se refere ao estado civil teve maior representação de mulheres casadas ou em união estável, $78,90 \%$.

Seguindo a análise tratando-se do número de filhos, $61,40 \%$ das puérperas já tinham de 1 a 2 filhos. No que diz respeito ao grau de instrução acadêmica, houve predomino de puérperas com o ensino médio completo ou incompleto com $61,40 \%$ da amostra.

No quesito profissão houve maior abrangência das que trabalham no lar, com 50,90\%. Com relação à renda mensal observou-se que $59,60 \%$ da amostra era composta por puérperas que recebem de 1 a 3 salários mínimos, seguidas das que não têm renda fixa ou que recebem menos de 1 salário mínimo com $35 \%$ da população estudada.

$\mathrm{Na}$ Tabela 2 refere-se aos fatores analisados pela escala de Escore de Creasy, que utiliza escores para avaliar 0 risco aumentado para o parto prematuro e tem como critérios que foram definidos, determinados e validados por este: 1. Baixo risco - escore de 0 - 5 pontos; 2. Médio risco - escore de 6 - 9 pontos e 3 . Alto risco - escore de 10 ou mais pontos. A tabela 2, também trás os dados coletados das 57 puérperas com Recém Nascido Pré-Termo (RNPT), e a porcentagem 
de cada fator com relação ao total.

Tabela 2 - Fatores avaliados quanto a Escala de Creasy de puérperas atendidas na maternidade de referência Estadual. Teresina-PI, 2014.

\begin{tabular}{lcc}
\hline \multicolumn{1}{c}{ FATORES AVALIADOS NO } & NÚMERO & PORCENTAGEM \\
\multicolumn{1}{c}{ SCORE DE CREASY * } & & \\
\hline CONDIÇÃO SOCIOECONÔMICA & & \\
Crianças em casa & 15 & $26,31 \%$ \\
Baixo nível socioeconômico & 16 & $28,07 \%$ \\
$<18$ anos & 7 & $12,28 \%$ \\
< 20 anos e >40 anos & 13 & $22,79 \%$ \\
Nível socioeconômico MUITO BAIXO & 24 & $42,10 \%$ \\
Peso < 45 kg & 7 & $12,28 \%$ \\
Estatura < 1,5m & 9 & $15,78 \%$ \\
HISTÓRIA MATERNA PRÉVIA & & \\
Aborto prévio & & \\
Prematuro prévio & 8 & $14,03 \%$ \\
HÁBITOS MATERNOS & 7 & $12,28 \%$ \\
Trabalha fora do lar & & $24.56 \%$ \\
Trabalho Extenuante & 14 & $19,29 \%$ \\
Muito tempo do translado casa - trabalho & 11 & $14,03 \%$ \\
ATUAL GESTAÇÃO & 8 & \\
< 7 kg de ganho ponderal com 32 semanas & & $22,80 \%$ \\
Hipertensão & 13 & $36,84 \%$ \\
Bacteriúria & 21 & $31,57 \%$ \\
Metrorragia após 12 semanas de gestação & 18 & $19,29 \%$ \\
Placenta prévia & 11 & $3,50 \%$ \\
\hline
\end{tabular}

* Pode estar presente mais de um fator.

Foi observado que de acordo com a escala de Escore de Creasy que utiliza escores para avaliar o risco aumentado para o parto prematuro, analisou-se segundo os critérios de classificação que das 57 puérperas entrevistadas 17 apresentaram baixo risco (escore de 0 - 5 pontos); 17 médio risco (escore de 6 - 9 pontos) e 23 alto risco (escore de 10 ou mais pontos).

De acordo com o escore, observou-se que as características mais relevantes quanto ao status socioeconômico foram à presença de um nível socioeconômico muito baixo, com $42,10 \%$ das puérperas entrevistadas, seguido de baixo nível socioeconômico com $28,07 \%$ das mesmas, e o fator ter crianças em casa com $26,31 \%$. Notou-se ainda que a idade menor de 20 anos e maior de 40 anos foi um fator comum entre as puérperas que tiveram RNPT com 22,79\%.

Quanto à história materna prévia, observou-se que 12,28\% das mulheres já tiveram parto prematuro prévio, e $14,03 \%$ aborto prévio. Sobre os hábitos maternos tem-se que as mulheres que trabalham fora do lar somam $24,56 \%$ das puérperas entrevistadas, e que $19,29 \%$ dessas tinham trabalho extenuante.

No que se refere a atual gestação, observou-se que 36,84\% das puérperas tiveram hipertensão arterial sistêmica durante a gestação, que 31,57\% tiveram Bacteriúria, e que 19,29\% apresentaram Metrorragia após 12 semanas de gestação.

Durante a realização do estudo pôde-se observar patologias que foram encontradas com frequência nas puérperas que tiveram RNPT, sendo elas Pré-eclâmpsia, Eclâmpsia, Oligodramnia, Aminiorrexe Prematura, Descolamento Prematuro de Placenta e Vulvovaginite.

No que se refere às intervenções assistenciais para as gestantes visando evitar ou minimizar as chances do parto prematuro, observou-se que a minoria dos casos teve alguma intervenção, sendo encaminhadas para a maternidade de referência por risco de ter um RNPT, com apenas $15,79 \%$ da amostra. Já a maioria das puérperas que tiveram RNPT 
apresentou algum problema durante a gestação e foram encaminhadas para a maternidade no final da gestação onde ficaram internadas por algum tempo realizando intervenções para maior maturação fetal, ou realizaram de imediato 0 parto, contando com $57,90 \%$ do todo.

Algumas gestantes foram internadas em hospitais dos municípios no qual residem ou municípios próximos por complicações durante a gestação, aonde foram realizadas intervenções para prolongar a gestação, porém sem sucesso o que levou ao encaminhamento das mesmas para a maternidade de referência, sendo estas representadas por 14,03\% dos casos, houve acontecimentos da gestante ser encaminhada para a maternidade de referência apenas após ter 0 RNPT, contando com $5,26 \%$ das ocorrências, e ainda eventos nos quais a gestante chegava à maternidade de referência por conta própria ou por indicação de um amigo/conhecido, e não pelo encaminhamento médico que seria uma conduta correta por parte da equipe de Pré-natal, representando 7,02\% dos fatos.

Entre as interações medicamentosas em potencial analisadas, observou-se que o número médio de interações por paciente foi de 2,51 $\pm 2,58$. Essas interações também estão relacionadas à presença de outras doenças, associadas à AR, como a Hipertensão Arterial Sistêmica (HAS), relatada por $14(45,16 \%)$ pacientes, e depressão (12,90\%).

A associação entre a média de medicamentos utilizados por paciente e a média das interações em potencial foi significativa $(p=0,001104)$, sendo possível verificar que quanto mais medicamentos são administrados, maiores são as chances de ocorrer interações entre eles. As interações medicamentosas presentes neste estudo estão descritas na Tabela 4.

\section{DISCUSSÃO}

Quanto ao perfil das puérperas que tiveram RNPT, observou-se que à idade predominante foi da faixa etária de 19 a 25 anos (35,9\%), seguida das mulheres com idade de 26 a 32 anos (24,56\% da amostra) e de 12 a 18 anos (21,05\%), e demais de 33 a 40 anos ou mais (19,30\%).

Estudos mostram que em relação à idade materna, a maioria dos casos de RNPT ocorre na adolescência, sendo que a média encontrada neste estudo foi de $21,05 \%$, menor do que a média encontrada por outros autores, como no estudo realizado em Guarapuava, PR, que mostra que 30\% das mães de prematuros se encontravam na faixa etária entre 15 e 19 anos$^{2}$.

No que concerne ao estado civil da mãe, um estudo mostra que houve uma maior prevalência de prematuridade entre as viúvas, e uma menor prevalência entre as mães casadas contrastando com a realidade do presente estudo no qual teve maior representatividade entre as mulheres casadas ou em união estável, com 78,94\% dos casos ${ }^{7}$.

Quanto a gestações prévias, observou-se que as mulheres primíparas tiveram um aumento de duas vezes nas chances de prematuridade ${ }^{8}$, corroborando com este estudo que mostrou que $61,40 \%$ das puérperas eram primíparas.

A baixa escolaridade é um fator associado a situações de risco para a mãe e o RN, pois impede 0 acesso a informações e orientações, dificultando o exercício dos direitos e da cidadania. Pois o nível de escolaridade interfere nas condições de vida e saúde das pessoas e que quanto menor a escolaridade, maior é a dificuldade de entendimento da importância de cuidados inerentes ao período gestacional, o que pode levar a um pré-natal de início tardio, a uma alimentação inadequada, e ainda a hábitos que prejudicam a gestação². No que diz respeito ao grau de instrução encontrado nesse estudo, houve predomino de puérperas no ensino médio incompleto ou completo $(61,40 \%)$, seguido de ensino fundamental incompleto correspondendo a $26,30 \%$ da amostra.

A maioria das mães que tiveram seus filhos prematuros não tinha atividade profissional, eram do lar8, corroborando mais uma vez com este estudo em que houve maior abrangência de mulheres que exerciam atividades somente no lar, com $50,90 \%$ das participantes. Sobre a renda mensal observou-se que $59,60 \%$ das puérperas recebem de 1 a 3 salários mínimos, seguidas das que não têm renda fixa ou que recebem menos de 1 salário mínimo com 35\% da população estudada, o que mostra uma renda relativamente baixa.

Outro ponto estudado foi quanto às patologias, observou-se nas puérperas que tiveram RNPT, a presença de pré-eclâmpsia, eclâmpsia, oligodramnia, aminiorrexe prematura, descolamento prematuro de placenta e vulvovaginite. No estudo sobre "As complicações obstétricas de maior incidência observadas em achados na literatura" registrou-se, a doença hipertensiva específica da gestação (DHEG), que ocorreu em $8,1 \%$ das puérperas, e a hiperêmese que ocorreu 
em 2,7\% das mulheres estudadas 9 .

Percebe-se que vários são os fatores de risco que podem levar a um parto prematuro, exercendo importante contribuição para tal acontecimento, onde o acompanhamento e orientações adequados para a mulher durante a gestação devem acontecer através do acompanhamento do pré-natal, detectando precocemente as mulheres com riscos favoráveis para ter um futuro RNPT.

Com base no estudo pode-se dizer que uma forma de evitar o parto prematuro, é ter conhecimento aprofundando do perfil das mulheres gravidas, abordando com mais intensidade cada uma das variáveis que tiveram efeito na investigação aqui realizada, desde o perfil socioeconômico, história e hábitos maternos, bem como da atual gestação, trabalhando na formulação de estratégias mais específicas para estes grupos. Outro fator importante e que se constata é a necessidade de uma rede de atenção à mulher para que sejam encaminhadas adequadamente e os riscos e de partos prematuros sejam diminuídos, evitados ou então, tenham uma assistência adequada para minimizar os óbitos neste período.

Importante também que, novas pesquisas existam como forma de dar mais subsídios e fundamentação para os resultados aqui encontrados, analisando a realidade de outros locais, confirmando ou achando novos resultados, causando impacto de ser capaz de induzir mudanças de comportamento e de assistência nos órgãos públicos, e, sobretudo uma atenção mais assídua das equipes de saúde que acompanham as gestantes, apresentando decisões mais apropriadas desde o inicio da gestação das mulheres que tenham fatores de risco para o parto prematuro, como forma de evitar ou minimizar tal acontecimento e suas consequências tanto para mãe como para a criança.

\section{REFERÊNCIAS}

1 - Brasil. Ministério Da Saúde. Portal Brasil [Internet]. Tipos de parto. Brasília (DF): Ministério da Saúde; 2013. [citado 2014 Jun 7]. Disponível em: <http://www.brasil.gov.br/sobre/saude/maternidade/parto/os-tipos-de-parto>.

2 - Ramos HAC, Cuman RKN. Fatores de risco para prematuridade: pesquisa documental. Esc. Anna Nery, Rio de Janeiro - RJ. 2009; 13 (2): 297-304.

3 - Tavares RFS. Sala de parto para o pediatra. Revinter, Rio de Janeiro - RJ. 2005.

4 - Vianna M. Parto prematuro/prevenção. Femina, Rio de Janeiro - RJ. 2006; 34 (11): 731 - 734.

5 - Federação Brasileira das Associações de Ginecologia e Obstetrícia. Manual de Orientação Gestação de Alto Risco. FEBRASGO, 2011. [citado 2014 Ago 14]. Disponível em: <https://pt.scribd.com/doc/131196255/88962406Manual-Gestacao-Alto-Risco-2011-pdf>.

6- Gil AC. Como elaborar projetos de pesquisa. 4 ed. São Paulo: Atlas; 2008.

7- Cascaes AM; Gaúche H; Baramarchi FM; Borges CM; Peres KG. Prematuridade e fatores associados no Estado de Santa Catarina, Brasil, no ano de 2005: análise dos dados do Sistema de Informações sobre Nascidos Vivos. Cad. Saúde Pública, Rio de Janeiro - RJ. 2008; 24(5):1024-1032.

8 - Almeida AC; Jesus ACP; Lima PFT; Araújo MFM; Araújo TM. Fatores de risco maternos para prematuridade em uma maternidade pública de Imperatriz- MA. Rev Gaúcha Enferm. Porto Alegre - RS. 2012; 33(2):86-94.

9 - Souza NL, Araújo ACPF, Costa ICC. Representações sociais de puérperas sobre as síndromes hipertensivas da gravidez e nascimento prematuro. Rev. Latino-Am. Enfermagem. 2013; 21(3): 726-733. [citado 2014 Set 03]. Disponível em: <http://dx.doi.org/10.1590/S0104-11692013000300011>. 
12. Miranda ARA, Araújo CS; Faleiros CFS. Diagnósticos de enfermagem na reabilitação de adultos com artrite reumatóide, segundo Roy e a CIPE®. Rev Enferm do Centro-Oeste Mineiro. 2013; 2:314-25.

13. Helmick CG, Felson DT, Lawrence RC, Gabriel S, Hirsch R, Kwoh CK et al. Estimates of the prevalence of arthritis and other rheumatic conditions in the United States: Part I. Arthritis \& Rheumatism. 2008; 58:15-25.

14. Senna ER, Barros AL, Silva EO, Costa IF, Pereira LV, Ciconelli RM et al. Prevalence of rheumatic diseases in Brazil: a study using the copcord approach. J Rheumatol. 2004; 31(3):594-7.

15. Boletim da Sociedade Brasileira de Reumatologia. Rev Bras Reumatol. 2014 Jan/Fev/Mar; (5), XXXVIII. [citado em 2015 maio 05]. Disponível em: http://reumatologia.com.br/PDFs/boletimSBR_JanFevMar_2014.pdf.

16. Moura MC, Zakszewski PTS, Silva MBG, Skare TL. Perfil dos pacientes com manifestações extraarticulares de artrite reumatoide de um serviço ambulatorial em Curitiba, Sul do Brasil. Rev Bras Reumatol. 2012; 52(2):679-94.

17. Souza OMN. Avaliação demográfica, clínico-laboratorial e genética de indivíduos com lúpus eritematoso sistêmico e artrite reumatóide residentes em região tropical. Dissertação (Mestrado em Bioquímica; Biologia Molecular) - Universidade Federal do Rio Grande do Norte, Natal: UFRN; 2006. 99 p.

18. IBGE. Instituto Brasileiro de Geografia e Estatística. Censo demográfico. Características da População e dos Domicílios: Resultados do Universo. Rio de Janeiro: IBGE; 2010.

19. Bengtsson C, Nordmark B, Klareskog L, Lundberg I, Alfredsson L, EIRA Study Group. Socioeconomic status and the risk of developing rheumatoid arthritis: results from the Swedish EIRA Study. Ann Rheum Dis. 2005; 64(11):1588-94.

20. Schmidt MI, Duncan BB, Azevedo e Silva G, Menezes AM, Monteiro CA, Barreto SM. Chronic non communicable diseases in Brazil: burden and current challenges. Lancet. 2011; 377(9781):1949-1961.

21. Rojas VA, Diaz FJ, Calvo PE, Salazar JC, Iglesias GA, Mantilla RD et al. Familial disease, the HLADRB1 shared epitope and anti-CCP antibodies influence time at appearance of substantial joint damage in rheumatoid arthritis. J Autoimmun. 2009; 32(1):64-9.

22. Harney S, Wordsworth BP. Genetic epidemiology of rheumatoid arthritis. Tissue Antigens. 2002; 60:46573.

23. Mota LMH, Cruz BA, Brenol CV, Pereira IA, Rezende-Fronza LS, Bertolo MB et al. Atualização do consenso brasileiro no diagnóstico e tratamento da artrite reumatoide. Rev Bras Reumatol. 2012; 52(2):135-74.

24. Firestein GS. "Etiology and pathogenesis of rheumatoid arthritis". J.R. O'Dell (Eds.), Kelley's Textbook of Rheumatology II. Elsevier Saunders, Philadelphia. 2013; 9:1059-1108.

25. Marion CE, Balfe LM. Potential advantages of interprofessional care in rheumatoid arthritis. J Manag Care Pharm. 2011; 17:25-29.

26. Louzada PJ, Souza BDB, Toledo RA, Ciconelli, RM. Análise descritiva das características demográficas e clínicas de pacientes com artrite reumatoide no Estado de São Paulo, Brasil. Rev Bras Reumatol. 2007; 47(2):84-90. 
27. Arrais PSD, Brito LL, Barreto ML, Coelho HLL. Prevalência e fatores determinantes do consumo de medicamentos no Município de Fortaleza, Ceará, Brasil Prevalence and determinants of medicines consumption in Fortaleza, Ceará, Brazil. Cad. Saúde Públ. 2005; 21(6):1737-46.

28. Filho JMC, Marcopito LF, Castelo A. Perfil de utilização de medicamentos por idosos em área urbana do Nordeste do Brasil. Rev Saúde Públ. 2004; 38:557-564.

29. Figueiras A, Caamaño F, Gestal-Otero JJ. Sociodemographic factors related to self-medication in Spain. Eur J Epidemiol. 2000; 16(1):19-26.

30. Malottki K, Barton PTA, Uthman AO, Liu Z, Routh K, Connock M, Moore D, Fry-Smith A, Chen YF. Adalimumab, eta?nercept, infliximab, rituximab and abaาtacept for the treatment of rheumatoid arthritis after the failure of a tumour necrosis factor inhibitor: a systematic review and economic evaluation. Heal $\neg$ th Technol Assess. 2011; 15:1-278.

31. Helin SA, Huupponen R, Virtanen A, Lammela J, Klaukka T. Frequent prescribing of drugs with potential gastrointestinal toxicity among continuous users of nonsteroidal anti-inflammatory drugs. Eur Clin Pharmacol. 2005; 61(5):425-31.

32. Bosscher K, Haegeman G, Elewaut D. Targeting inflammation using selective glucocorticoid receptor. Current Opinion in Pharmacology. Oxford; 2010; 10(4):497-504.

33. Anti SMA, Giorgi RDN, Chahade WH. Anti-inflamatórios hormonais: glicocorticóides. Einstein. 2008; 6(1):159-65.

34. Brasil. Ministério da Saúde. Portaria $n^{0} 710$, de 27 de junho de 2013. Aprova o Protocolo Clínico e Diretrizes Terapêuticas da Artrite Reumatoide . [citado em 2015 maio 01]. Disponível em: http://portalsaude.saude.gov.br/ images/pdf/2014/marco/07/ pcdt-artrite-reumatoide- 2013-retificado-2014.pdf.

35. Hazlewood GS, Barnabe CCM, Tomlinson GA, Marshall D, Bombardier C. The comparative efficacy and toxicity of initial disease-modifying anti-rheumatic drug choices for patients with moderate-severe early rheumatoid arthritis: A bayesian network meta-analysis. Arthritis and Rheumatism. 2011; 63(10):868.

36. Gaujoux VC, Smolen JS, Landewe R, Dougados M, Kvien TK, Mola EM et al. Current evidence for the management of rheumatoid arthritis with synthetic disease-modifying antirheumatic drugs: a systematic literature review informing the EULAR recommendations for the management of rheumatoid arthritis. Ann Rheum Dis. 2010; 69(6):1004-9.

37. Karalliedde L, Clarke SFJ, Lollignon U, Karalliedde J. Interações medicamentosas adversas. Rio de Janeiro: Guanabara Koogan; 2012.

38. Bezabeh S, Mackey AC, Kluetz P, Jappar D, Korvick J. Accumulating evidence for a drug-drug interaction between methotrexate and proton pump inhibitors. The oncologist. 2012; 17(4):550-4.

39. Lacy CF, Armstrong LL, Goldman MP, Lance LL. Drug Information Handbook. 20 ed. Hudson: LexiComp; 2011.

40. Chan A, Ko Y, Wong CM. Clinically Significant Drug-Drug Interactions Between Oral Anticancer Agents and Nonanticancer Agents: Profiling and Comparison of Two Drug Compendia. Ann Pharmacother. 2008; 42:1737-1748.

41. Maeda A, Tsuruoka S, Kanai Y, Endou H, Saito K, Miyamoto E, Fujimura A. Evaluation of the interaction between nonsteroidal anti-inflammatory drugs and methotrexate using human organic anion transporter 3-transfected 
cells. Eur J Pharmacol. 2008; 596(1):166-172.

42. Silva JED. Interação medicamentosa entre anti-inflamatórios não esteroides e anti-hipertensivo em pacientes hipertensos internados em um hospital público: uma abordagem em farmacovigilância. Rev Baiana Saúde Públ. 2008; 32(1):18-28.

43. Fortes ZB, Nigro D. Aspectos farmacológicos da interação anti-hipertensivos e anti-inflamatórios não esteroides. Rev Bras Hipertens. 2005; 12(2):108-11.

44. Bisognin M. Análise do Registro Brasileiro de Monitorização de Terapias Biológicas em Doenças Reumáticas dos pacientes com Artrite Reumatóide, atendidos no Serviço de Reumatologia do Hospital Universitário da UFMS. Santa Maria, RS; 2013.

45. Panoulas VF, Douglas KM, Stavropoulos KA, Metsios GS, Nightingale P, Kita MD et al. Long-term exposure to medium-dose glucocorticoid therapy associates with hypertension in patients with rheumatoid arthritis. Rheumatology. Oxford; 2008; 47(1):72-5.

46. Morrison A, Rosen RD, Van AJ, Watson DJ. Systematic review of trials of the effect of continued use of oral non-selective NSAIDs on blood pressure and hypertension. Current Medical Research and Opinion. 2007; 23(10):2395-2404.

47. Korolkovas A. Dicionário terapêutico. Rio de Janeiro: Guanabara Koogan; 2015.

48. Mion Jr, Kohlmann Jr, Machado CA, Amodeo C, Gomes MAM, Praxedes JN (2007). Sociedade Brasileira de Cardiologia, Sociedade Brasileira de Hipertensão, Sociedade Brasileira de Nefrologia. VI Diretrizes Brasileiras de Hipertensão. Arq Bras Cardiol. 2010; 95:1-51.

49. Malachias MVB. VII Diretrizes Brasileiras de Hipertensão Arterial, 2016; 107(3):1-82.

50. Micromedex® Healthcare Series. Greenwood Village (CO): Thomson Reuters (Healthcare) Inc.; 2015. [citado em 2015 maio 05]. Disponível em: http://micromedex.com/.

51. Silva P. Farmacologia. 8. ed. Rio de Janeiro: Guanabara Koogan; 2010.

52. Dario AB, Faraco HC, Gevaerd MS, Domenech SC. Alterações psicológicas e exercício físico em pacientes com artrite reumatoide. Motricidade. 2010; 6(3):21-30.

53. Mella LFB, Bértolo MB, Dalgalarrondo P. Depressive symptoms in rheumatoid arthritis patients. Rev Bras Psiquiatr. 2010; 32:257-263.

54. Mota LMHD, Cruz BA, Brenol CV, Pereira IA, Fronza LS, Bertolo MB et al. Diretrizes para o tratamento da artrite reumatoide. Rev Bras Reumatol. 2013; 53:158-183.

55. Kiely PD, Brown AK, Edwards CJ, O'Reilly DT, Ostör AJ, Quinn M et al. Contemporary treatment principles for early rheumatoid arthritis: a consensus statement. Rheumatology. Oxford; 2009; 48(7):765-772.

56. Brasil. Portaria $n^{0} 1.555$, de 30 de julho de 2013. Dispõe sobre as regras de financiamento e execução do Componente Especializado da Assistência Farmacêutica no âmbito do Sistema Único de Saúde (SUS). Brasília; 2013.

57. Brasil. Ministério da Saúde. Portaria SCTIE nº 66 de 06 de novembro de 2006. Protocolo Clínico e Diretrizes Terapêuticas - Artrite Reumatoide. [citado em 2015 maio 05]. Disponível em: http://www.portal.saude.gov.br/ 